\title{
DIGITALCOMMONS
}

8-1-1978

\section{Application of an extremum principle to the variational determination of the generalized oscillator strengths of helium}

\author{
J.M. Wadehra \\ New YorkUniversity, ad5541@wayne.edu \\ Larry Spruch \\ New York University \\ Robin Shakeshaft \\ Texas A\&M University
}

\section{Recommended Citation}

Wadehra JM, Spruch L, \& Shakeshaft R. Application of an extremum principle to the variational determination of the generalized oscillator strengths of helium. Phys. Rev. A. 1978;18(2):344-49. doi: 10.1103/PhysRevA.18.344

Available at: http://digitalcommons.wayne.edu/phy_astro_frp/75 


\title{
Application of an extremum principle to the variational determination of the generalized oscillator strengths of helium
}

\author{
J. M. Wadehra* and Larry Spruch \\ Physics Department, New York University, New York, New York 10003 \\ Robin Shakeshaft \\ Physics Department, Texas A\&M University, College Station, Texas 77843
}

(Received 16 September 1977)

\begin{abstract}
Variational principles have been used extensively for estimating some given functional $F(\phi, \phi \dagger)$ where the functions $\phi$ and $\phi^{\dagger}$ are well defined by a set of differential equations and boundary conditions but cannot be determined exactly. The variational principle for the estimation of a matrix element of an arbitrary Hermitian operator $W$ involves not only the trial wave functions $\phi_{t}$ but also trial auxiliary Lagrange functions $L_{t}$; the $L_{t}$ depend on the $\phi_{t}$ and on $W$. To determine the parameters in the $L_{t}$ efficiently, a functional $M\left(L_{t t}\right)$ is constructed which is an extremum for $L_{t t}=L_{t}$. The technique was recently used successfully in the variational estimation of two diagonal matrix elements. We here use this technique for the variational estimation of an off-diagonal matrix element, the generalized oscillator strengths of helium for the transition between the ground state and the excited $2^{1} P$ state. Two $L_{t}$ 's must be determined. Our results on helium indicate that variational estimates are a significant improvement over the first-order estimates. The results are also compared with those obtained nonvariationally using more elaborate groundand excited-state wave functions; the comparison represents a check on the method. It is not yet clear which of the two approaches is more efficient.
\end{abstract}

\section{INTRODUCTION}

The diagonal matrix element of a Hermitian operator $W$ with respect to the lowest state of some given symmetry is usually estimated by first evaluating the variational parameters contained in a trial wave function $\phi_{t}$ by a RayleighRitz energy minimization procedure, and then evaluating the matrix element by using $\phi_{t}$. For off-diagonal matrix elements for which each of the two wave functions is associated with a state which is the lowest state of a given symmetry, each of the two trial functions is obtained by a Rayleigh-Ritz method. (For higher states, one would use the generalization of the Rayleigh-Ritz procedure, the Hylleraas-Undheim approach.) However, the estimate of a matrix element evaluated by such a procedure is not comparable in accuracy with the energy estimate obtained in a Rayleigh-Ritz calculation.

An alternative procedure is to use a variational principle to estimate the relevant matrix element; by definition a variational principle gives an estimate good to second order in the error in $\phi_{t}$, which is of course the accuracy of the energy estimate obtained in a Rayleigh-Ritz calculation. A variational estimate of a bound-state matrix element of an arbitrary Hermitian linear operator can be constructed from trial normalized boundstate wave functions and certain auxiliary functions., 2 (More generally, ${ }^{3}$ one can construct a variational principle for just about any entity of interest in mathematical physics, with the auxiliary functions particular examples of Lagrange undetermined multipliers-which can be a constant $\lambda$, a function $L$, an operator $\Lambda, \ldots$ ). A Lagrange undetermined multiplier function $L$ is well defined by an inhomogeneous differential equation and appropriate boundary conditions. One need not obtain the $L$ 's exactly to maintain the variational principle; one need "merely" obtain an estimate $L_{t}$ good to first order. One can obtain an adequate estimate $L_{t}$ for a very restricted class of $\phi_{t}$ 's-a Hartree product, for example. If, however, one seeks an accuracy greater than that thereby achieved, one would like to introduce a general $\phi_{t}$; but a near singularity then arises in the inhomogeneous differential equation that had been used as the defining equation for $L_{t}$ for all but some very special cases of the wave functions that appear in the matrix elements, and the variational principle is destroyed. This difficulty has recently been circumvented. ${ }^{4}$ With an appropriately modified equation defining $L_{t}$, one constructs a functional $M\left(L_{t t}\right)$ which is an extremum for $L_{t t}$ $=L_{t}$. The minimization of $M$ determines the parameters contained in $L_{t t}$, an approximation to $L_{t}$ (which is, in turn, an approximation to $L$ ).

This technique was recently used successfully in the variational estimation of two diagonal matrix elements, namely, the diamagnetic susceptibility and the form factor of the helium atom in its ground state. ${ }^{5}$ In the present work, we have used this technique for the variational estimation of an 
off-diagonal matrix element, the generalized oscillator strengths of helium for the transition between the ground state and the excited $2^{1} P$ state. The results of the present work represent the first effort to estimate variationally an off-diagonal matrix element using an extremum principle. Such an estimation requires the determination of two $L_{t}$ 's, which can be determined independently.

(For the particular matrix element involved, the commutation trick used by Schwartz ${ }^{1}$ should be free of singularities; his procedure works for nodeless functions, and the $1^{1} S$ ground state is known to be nodeless, and the procedure can probably be adapted to the $2^{1} P$ state. However, his. procedure can surely not be used for matrix elements involving excited ${ }^{1} S$ and/or higher ${ }^{1} P$ states. The extremum principle procedure, ${ }^{4}$ however, is always free of near singularities; we have chosen to consider the transition between the lowest ${ }^{1} S$ and ${ }^{1} P$ states because we are interested in studying the applicability of the extremum principle formulation of a variational principle to an offdiagonal matrix element, and we therefore chose a relatively simple case.)

The generalized oscillator strength of the helium atom for the transition from the ground state (wave function $\phi_{1}$, energy $E_{1}$ ) to the excited state (wave function $\phi_{2}$, energy $E_{2}$ ) is defined as

$$
f\left(k^{2}\right)=\frac{E_{2}-E_{1}}{\left(e^{2} / 2 a_{0}\right)\left(k a_{0}\right)^{2}}\left|\left\langle\phi_{2}\left|W^{\prime}\right| \phi_{1}\right\rangle\right|^{2},
$$

where

$$
W^{\prime}=\mathrm{e}^{i \overrightarrow{\mathrm{k}} \cdot \overrightarrow{\mathrm{r}}_{1}}+e^{i \overrightarrow{\mathrm{k}} \cdot \overrightarrow{\mathrm{r}}_{2}} .
$$

$\overrightarrow{\mathrm{k}} \hbar$ is the change in momentum of the fast incident electron undergoing inelastic collision with the helium atom. $\overrightarrow{\mathrm{r}}_{1}$ and $\overrightarrow{\mathrm{r}}_{2}$ are the coordinates of the two bound electrons.

\section{THE EXTREMUM PRINCIPLE}

In this section we outline the extremum principle applicable to the variational estimation of the off-diagonal matrix element of interest. A complete discussion of the general case, along with the detailed derivations, can be found elsewhere..$^{3,4}$

Let $\phi_{1 t}$ and $\phi_{2 t}$ be normalized approximations to $\phi_{1}$ and $\phi_{2}$, the true normalized ground-state and the lowest ${ }^{1} P$ excited-state wave functions, respectively, and let $E_{1 t}$ and $E_{2 t}$ be the corresponding variational estimates of $E_{1}$ and $E_{2}$, the true ground- and excited-state energies, respectively. We are interested in obtaining a variational estimate of the matrix element $\langle W\rangle=\phi_{1}^{\dagger} W \phi_{2}$ for a linear Hermitian operator $W$. Note that the trial estimate

$$
\langle W\rangle_{t}=\phi_{1 t}^{\dagger} W \phi_{2 t}
$$

differs from the true value $\langle W\rangle$ by first order in $\delta \phi_{1} \equiv \phi_{1 t}-\phi_{1}$ and in $\delta \phi_{2} \equiv \phi_{2 t}-\phi_{2}$.

Although we are ultimately interested in $|\langle W\rangle|^{2}$, we are here concerned with $\langle W\rangle$ itself, and one cannot construct a variational principle for $\langle W\rangle$ unless $\langle W\rangle$ has been uniquely defined. It will therefore be necessary to define the phase of $\langle W\rangle$. This can be done by defining the phases of both $\phi_{1}$ and $\phi_{2}$, or simply their relative phase. We will return to this point in Sec. III.

By construction, the variational estimate $\langle W\rangle_{v}$ differs from $\langle W\rangle$ only in second-order terms; it is given by ${ }^{1-3}$

$$
\begin{aligned}
\langle W\rangle_{v} & =\langle W\rangle_{t}+2 L_{1 t}^{\dagger}\left(H-E_{1 t}\right) \phi_{1 t}+2 L_{2 t}^{\dagger}\left(H-E_{2 t}\right) \phi_{2 t} \\
& \equiv\langle W\rangle_{t}+\Delta_{1 t}+\Delta_{2 t},
\end{aligned}
$$

where $L_{1 t}$ and $L_{2 t}$ are solutions of the inhomogeneous differential equations

$$
\begin{aligned}
& \left(H_{\bmod , t}^{(1)}-E_{1 t}\right) L_{1 t}=-\frac{1}{2} W \phi_{2 t}, \\
& \left(H_{\bmod , t}^{(2)}-E_{2 t}\right) L_{2 t}=-\frac{1}{2} W \phi_{1 t} ;
\end{aligned}
$$

the trial modified Hamiltonians are defined by

$$
H_{\mathrm{mod}, t}^{(n)}=H-\sum_{i=1}^{n} \frac{H \phi_{i t} \phi_{i t}^{\dagger} H}{E_{i t}} .
$$

$\Delta_{1 t}$ and $\Delta_{2 t}$ are first-order corrections that cancel the first-order terms in $\langle W\rangle_{t}$ which arise due to the approximate nature of $\phi_{1 t}$ and $\phi_{2 t}$, respectively. We note that two requirements for the positivity of the operators $H_{\mathrm{mod}, t}^{(i)}-E_{i t}$ for $i=1$ and 2 , which are essential for the extremum principle, ${ }^{4}$ namely, that $\left(\phi_{1 t}, \phi_{2 t}\right)=0$ and $\left(\phi_{1 t}, H \phi_{2 t}\right)=0$, are trivially satisfied in the present context since $\phi_{1 t}$ and $\phi_{2 t}$ are states of different total orbital angular momenta.

$L_{1 t}$ and $L_{2 t}$ are uniquely defined by Eqs. (2.3) and the requirement ${ }^{3}$ of quadratic integrability, since the corresponding homogeneous equations have no solutions. In particular, the boundary conditions on $L_{1 t}$ and $L_{2 t}$ are obtained by projecting Eqs. (2.3a) and (2.3b) onto $\phi_{1 t}$ and $\phi_{2 t}$, respectively; one finds

$$
\phi_{1 t}^{\dagger} L_{1 t}=\frac{1}{2}\langle W\rangle_{t} / E_{1 t}, \quad \phi_{2 t}^{\dagger} L_{2 t}=\frac{1}{2}\langle W\rangle_{t} / E_{2 t} .
$$

These conditions provide a useful numerical check on the results obtained for $L_{1 t t}$ and $L_{2 t t}$, respectively, but the checks can be satisfied even if important components of $L_{1 t t}$ and $L_{2 t t}$ are very much in error. ${ }^{5}$

It was shown ${ }^{4}$ that the operator $\left(H_{\bmod , t}^{(n)}-E_{n t}\right)$ is positive definite provided the inequality

$$
E_{\mathrm{nt}} \leqslant \prod_{i=1}^{n}\left(\frac{E_{i}}{E_{i t}}\right) \times E_{n+1}
$$

holds. This is always possible in principle for 
sufficiently accurate wave functions, and in the present context poses no difficulties at all in practice. Thus $L_{1 t t}$ and $L_{2 t t}$ can be obtained by minimization of the functionals

$M_{11}\left(L_{1 t t}\right)=L_{1 t t}^{\dagger}\left(H_{\mathrm{mod}, t}^{(1)}-E_{1 t}\right) L_{1 t t}+L_{1 t t}^{\dagger} W \phi_{2 t}$

and

$M_{22}\left(L_{2 t t}\right)=L_{2 t t}^{\dagger}\left(H_{\bmod , t}^{(2)}-E_{2 t}\right) L_{2 t t}+L_{2 t t}^{\dagger} W \phi_{1 t}$,

respectively, with respect to the variational parameters contained in the particular choices of $L_{1 t t}$ and $L_{2 t t}$.

\section{RESULTS}

To define $\langle W\rangle$ uniquely, we take $\phi_{1}$ to be real and we take $\phi_{2}$ to be of the form

$$
\phi_{2}=F\left(r_{1}, r_{2}, r_{12}\right) Y_{10}\left(\hat{r}_{1}\right)+1-2 \text {, }
$$

with $F$ real.

For $\phi_{1 t}$ we chose the real 20-parameter wave function of Hart and Herzberg. ${ }^{6}$ For the $2^{1} P$ excited state of helium, we determined a 20-parameter wave function of the form

$\phi_{2 t}=r_{1} f\left(r_{1}, r_{2}, r_{12}\right) e^{-\left(\alpha r_{1}+\beta r_{2}\right) / 2} Y_{10}\left(\hat{r}_{1}\right)+1-2$

with

$$
f\left(r_{1}, r_{2}, r_{12}\right)=\sum C_{t m n} r_{12}^{l} r_{1}^{m} r_{2}^{n}
$$

$l, m$, and $n$ are non-negative integers and $l+m+n$ $\leqslant 3 ; \alpha$ and $\beta$ are nonlinear variational parameters; and the $C_{l m n}$ are linear variational parameters. Table I gives an indication of the accuracy of the wave functions. Inequality (2.6) was always satisfied, ensuring the positivity of $\left(H_{\bmod , t}^{(n)}-E_{n t}\right)$. Table II shows the generalized oscillator strengths of helium for a transition between the ground state and the $2^{1} P$ excited state, for a few values of $k^{2} a_{0}^{2}$, using the above $\phi_{1 t}$ and $\phi_{2 t}$. These results are also compared with the results of Kim and Inokuti, ${ }^{7}$ which were obtained with more elaborate trial functions, containing 53 and 52 linear parameters, respectively. Neither of these sets of results is variational. (Our variational results will be given later.)

The choice of the form of $L_{1 t t}$ and $L_{2 t t}$ was made as follows. The Hamiltonian of the helium atom is

$$
H=-\frac{\hbar^{2}}{2 m}\left(\nabla_{1}^{2}+\nabla_{2}^{2}\right)-2\left(\frac{e^{2}}{r_{1}}+\frac{e^{2}}{r_{2}}\right)+\frac{e^{2}}{r_{12}}
$$

The dependence of the wave functions on the Eulerian angles - the angles which define the normal to and the angle of rotation of the triangle determined by $r_{1}, r_{2}$, and $r_{12}$-is determined completely by angular momentum, parity, and symmetry. In particular, $\phi_{1}$ is a function $r_{1}, r_{2}$, and $r_{12}$ only, while $\phi_{2}$ also depends on the Eulerian angles [see Eq. (3.1)]. On the other hand, the dependence of the auxiliary functions on the Eulerian angles is determined also by the operator $W$ whose matrix elements are sought between the ground and excited states. The operator whose matrix elements are required is

$$
W^{\prime}=e^{i \overrightarrow{\mathrm{k}} \cdot \overrightarrow{\mathrm{r}}_{1}}+e^{i \overrightarrow{\mathrm{k}} \cdot \overrightarrow{\mathrm{r}}_{2}} .
$$

Note that $W^{\prime}$ is not Hermitian. However, to within an irrelevant factor of $i$, the matrix element of $W^{\prime}$ between $\phi_{2}$ and $\phi_{1}$ is the same as that of the Hermitian operator

$W \equiv(12 \pi)^{1 / 2}\left[j_{1}\left(k r_{1}\right) Y_{10}\left(\hat{r}_{1}\right)+j_{1}\left(k r_{2}\right) Y_{10}\left(\hat{r}_{2}\right)\right] ;$

$W$ is the component of unit total orbital angular momentum of $-i W^{\prime}$.

The angular dependences of $L_{1 t}$ and $L_{2 t}$ are determined by the operator $W$ and the angular dependence of the wave functions. From Eq. (2.3a), we note that $L_{1 t}$ has components of angular momentum zero and two. However the component of $L_{1 t}$ of angular momentum two does not contribute to the variational estimate, Eq. (2.2), of the matrix element and can be ignored. Thus we take $L_{1 t t}$ to be of the form

$L_{1 t t}=e^{-\gamma^{\prime}\left(r_{1}+r_{2}\right) / 2} \sum A_{l m n} r_{1}^{l} r_{2}^{l}\left(r_{1}^{m}+r_{2}^{m}\right) r_{12}^{n}$,

where $l, m$, and $n$ are non-negative integers.

Similarly Eq. (2.3b) suggests that $L_{2 t t}$ be chosen to be of the form

$$
\begin{aligned}
L_{2 t t}=\sum B_{l m n} & \left\{\left[r_{1}^{l+1} r_{2}^{m} e^{-\left(\alpha^{\prime} r_{1}+\beta^{\prime} r_{2}\right) / 2} Y_{10}\left(\hat{r}_{1}\right)\right]\right. \\
& +(1-2)\} r_{12}^{n},
\end{aligned}
$$

where, again, $l, m$, and $n$ are non-negative integers. $\alpha^{\prime}, \beta^{\prime}$, and $\gamma^{\prime}$ are nonlinear variational

TABLE I. Energy estimates in atomic units for the wave functions used.

\begin{tabular}{llccc}
\hline \hline Wave function & & $\begin{array}{c}\text { Accurate value } \\
\text { of energy }\end{array}$ & Energy & $|\delta E / E|$ \\
\hline Ground state & $1^{1} S$ & $-2.9037244^{\mathrm{c}}$ & $-2.9037179^{\mathrm{a}}$ & $2.24(-6)$ \\
Excited state & $2^{1} P$ & $-2.1238431^{\mathrm{c}}$ & $-2.1237954^{\mathrm{b}}$ & $2.25(-5)$ \\
\hline \hline
\end{tabular}

${ }^{\mathrm{a}}$ Reference 6 .

${ }^{\mathrm{c}}$ Reference 12.

${ }^{\mathrm{b}}$ Present work. 
TABLE II. Present values of the generalized oscillator strengths for $2{ }^{1} P$ excitations of helium (in a.u.) and the Kim-Inokuti values. Neither estimate is variational.

\begin{tabular}{rll}
\hline \hline$k^{2} a_{0}^{2}$ & Present value & Kim-Inokuti value ${ }^{\mathrm{a}}$ \\
\hline 0.1 & $2.3400(-1)$ & $2.3486(-1)$ \\
0.5 & $1.2816(-1)$ & $1.2852(-1)$ \\
2.2 & $1.6728(-2)$ & $1.6832(-2)$ \\
5.0 & $1.6294(-3)$ & $1.6655(-3)$ \\
10.0 & $1.0406(-4)$ & $1.1015(-4)$ \\
100.0 & $6.9587(-10)$ & $1.0565(-9)$ \\
\hline \hline
\end{tabular}

${ }^{a}$ Reference 7.

parameters and the $A_{l m n}$ and the $B_{l m n}$ are linear variational parameters.

In minimizing the functional $M_{11}$ with 7 and 13 linear parameters in $L_{1 t t}$, the nonlinear parameter $\gamma^{\prime}$ was varied in steps of 0.01 and with 20, 30,40 , and 45 parameters in $L_{1 t t}, \gamma^{\prime}$ was varied in steps of 0.05 . In the minimization of the functional $M_{22}$, the nonlinear parameters $\alpha^{\prime}$ and $\beta^{\prime}$ were always varied in steps of 0.1. Tables III and IV show the functionals $M_{11}, M_{22}$, the check on self-consistency, and the first-order corrections $\Delta_{1 t}$ and $\Delta_{2 t}$ to the matrix element as a function of the number of linear parameters for the particular case $\left(k a_{0}\right)^{2}=\mathbf{2 . 2}$.

In Table $\mathrm{V}$, we give the generalized oscillator strengths of helium for a few different values of $k^{2} a_{0}^{2}$. In calculating these values we used 45 parameters in $L_{1 t t}$ and 40 parameters in $L_{2 t t}$. These values of the generalized oscillator strengths should be among the most accurate theoretical values available at present.

\section{DISCUSSION}

The differential equations (2.3) for $L_{1 t}$ and $L_{2 t}$ are uncoupled so that each of these functions is estimated independently. Further, we notice from

TABLE III. Monotonic convergence of $M_{11}$ as a function of $N_{L_{1}}$, the number of linear parameters in $L_{1 t t}$, for $k^{2} a_{0}^{2}=2.2 . \Delta_{1 t}$ is the first-order correction to the matrix element [see Eq. (2.2)]. A partial check on the calculations is provided by a comparison of the values in the fourth column with the value $\phi_{1 t}^{\dagger} L_{1 t}=\frac{1}{2}\langle W\rangle_{t} / E_{1 t}$ $=-0.026449$.

\begin{tabular}{rcccc}
\hline \hline$N_{L_{1}}$ & $\gamma^{\prime}$ & $M_{11}$ & $\phi_{1 t}^{\dagger} L_{1 t t}$ & $\Delta_{1 t}$ \\
\hline 7 & 2.29 & -0.007379 & -0.025815 & $0.992(-5)$ \\
13 & 2.78 & -0.010599 & -0.026477 & $0.498(-5)$ \\
20 & 2.35 & -0.013087 & -0.026559 & $0.420(-4)$ \\
30 & 2.85 & -0.014113 & -0.026455 & $0.728(-4)$ \\
40 & 2.50 & -0.015318 & -0.026442 & $0.715(-4)$ \\
45 & 2.50 & -0.015545 & -0.026443 & $0.719(-4)$ \\
\hline \hline
\end{tabular}

TABLE IV. Monotonic convergence of $M_{22}$ as a function of $N_{L_{2}}$, the number of linear parameters in $L_{2 t t}$, for $k^{2} a_{0}^{2}=2.2 . \Delta_{2 t}$ is the first-order correction to the matrix element [see Eq. (2.2)]. A partial check on the calculations is provided by a comparison of the values in the fifth column with the value $\phi_{2 t}^{\dagger} L_{2 t}=\frac{1}{2}\langle W\rangle_{t} / E_{2 t}$ $=-0.036162$.

\begin{tabular}{cccccc}
\hline \hline$N_{L_{2}}$ & $\alpha^{\prime}$ & $\beta^{\prime}$ & $M_{22}$ & $\phi_{2 t}^{\dagger} L_{2 t t}$ & \multicolumn{1}{c}{$\Delta_{2 t}$} \\
\hline 10 & 1.4 & 3.8 & -0.224539 & -0.036471 & $-0.395(-5)$ \\
25 & 1.7 & 4.1 & -0.236958 & -0.036199 & $0.206(-3)$ \\
30 & 1.7 & 4.1 & -0.237464 & -0.036170 & $0.447(-3)$ \\
40 & 1.7 & 3.6 & -0.237534 & -0.036167 & $0.432(-3)$ \\
\hline \hline
\end{tabular}

the variational expression (2.2) for the matrix element that the auxiliary function $L_{1 t}$ corrects for the first-order error in $\phi_{1 t}$ and $L_{2 t}$ corrects for that in $\phi_{2 t}$. Table I suggests that $\phi_{1 t}$ is more accurate than $\phi_{2 t}$-it clearly is with regard to the estimate of the energy-so that we would generally expect the first-order correction $\Delta_{1 t}$ to be smaller than the first-order correction $\Delta_{2 t}$. This is consistent with the results in Table V.

Tables III and IV show the fulfillment of the conditions (2.5), but as noted above, these selfconsistency conditions do not provide any criterion for the accuracy of the estimates obtained of the value of the matrix element. Tables III and IV also exhibit the necessary monotonic decrease in value of the functionals; this too provides a check on the programming but does not provide any estimate of the accuracy of the matrix element. We can make two statements concerning that accuracy. Firstly, looking only at the entries $\Delta_{1 t}$ in Table III, it would seem that $\Delta_{1 t}$ has converged to within something of the order of $5 \times 10^{-6}$; similarly, looking only at the entries in Table IV under $\Delta_{2 t}$, it would seem that $\Delta_{2 t}$ has converged to within something like $1 \times 10^{-4}$. The errors just quoted are absolute errors. It follows that the matrix element estimate is probably accurate to roughly $\pm 1 \times 10^{-4}$. Second, we see from Table I that the energy estimates $E_{1 t}$ and $E_{2 t}$ are accurate to about two parts in 1000000 and two parts in 100000 , respectively. The accuracy of the variational estimate of the matrix element is limited therefore to roughly 2 parts in 100000 by the $\phi_{1 t}$ and $\phi_{2 t}$ chosen, no matter how elaborate a choice we make for $L_{1 t}$ and $L_{2 t}$. This is a relative error. Taking the value 0.1541065 for the matrix element for $k^{2} a_{0}^{2}=2.2$, a relative error of 2 parts in 100000 leads to an absolute error of roughly $3 \times 10^{-6}$ as the smallest possible error for the trial wave functions chosen. This is 100 times smaller than the absolute error of $1 \times 10^{-4}$ estimated above. The Kim-Inokuti value, incidentally, is 0.1540814 . This estimate is based on a more elaborate and 
TABLE V. The zeroth-order and variational estimate of the matrix element, $\langle W\rangle_{t}$ and $\langle W\rangle_{v}$, and the zeroth-order estimate $f_{t}\left(k^{2}\right)=2\left(E_{2}-E_{1}\right)\left|\langle W\rangle_{t}\right|^{2} / k^{2}$ (see Table II) and the variational estimate $f_{v}\left(k^{2}\right)=2\left(E_{2}-E_{1}\right)\left|\langle W\rangle_{v}\right|^{2} / k^{2}$ of the generalized oscillator strengths for different values of $k^{2}$, in atomic units.

\begin{tabular}{rccccccc}
\hline \hline \multicolumn{1}{c}{$k^{2}$} & $\langle W\rangle_{t}$ & $\Delta_{1 t}$ & $\Delta_{2 t}$ & $\langle W\rangle_{v}$ & $f_{v}\left(k^{2}\right)$ & $\begin{array}{c}\text { Kim-Inokuti } \\
\text { value of } \\
f\left(k^{2}\right)^{\mathrm{a}}\end{array}$ \\
\hline 2.2 & 0.1536024 & $0.7191(-4)$ & $0.4322(-3)$ & 0.1541065 & $1.6838(-2)$ & $1.6832(-2)$ \\
5.0 & 0.0722698 & $0.7408(-4)$ & $0.7924(-3)$ & 0.0731363 & $1.6687(-3)$ & $1.6655(-3)$ \\
10.0 & 0.0258286 & $0.4801(-4)$ & $0.7595(-3)$ & 0.0266361 & $1.1067(-4)$ & $1.1015(-4)$ \\
\hline \hline
\end{tabular}

${ }^{\mathrm{a}}$ Reference 7 .

better pair of trial functions than those used in the present calculation, for Kim and Inokuti used Weiss' wave functions, for which we calculate the fractional error $|\delta E / E|$ to be $0.24 \times 10^{-6}$ and 1.4 $\times 10^{-6}$ for the ground state and excited state, respectively. On the other hand, since their calculation is not variational, the relative error in their $\langle W\rangle$ is expected to be of the order of the larger of the two values of $|\delta E / E|^{1 / 2}$, namely, about $10^{-3}$. The absolute error in their $\langle W\rangle$ can therefore be expected to be roughly $10^{-4}$. The difference of $2 \times 10^{-5}$ between their estimate of $\langle W\rangle$ and ours is consistent with the above error estimates.

Preliminary attempts at an estimate of $\langle W\rangle$ for $\left(k a_{0}\right)^{2}=100$ indicated that the calculation would require considerably more effort than at lower values of $\left(k a_{0}\right)^{2}$ because of the difficulty in locating appropriate values of the nonlinear parameters. At high values of $\left(k a_{0}\right)^{2}$, one might try to obtain the leading term $L_{1 t}^{(0)}$ to $L_{1 t}$, for $L_{1 t}$ written as a power series in $1 / k$. If one could do so, one would then write $L_{1 t}$ as $L_{1 t}^{(0)}$ plus a term of the general form of Eq. (3.1). The additional form would not have much "physics" built into it, but the linear parameter it contains could be varied in the usual way, and one would only have to account for the difference between $L_{1 t}$ and $L_{1 t}^{(0)}$. One would proceed in an identical fashion in choosing $L_{2 t}$.

One can, of course, use the results of the present work to obtain variational bounds on the matrix element. ${ }^{8}$ A variational bound was, in fact, obtained for a diagonal matrix element, namely, the diamagnetic susceptibility. ${ }^{9}$ Alternatively, one could try to obtain an explicit value of the secondorder error in the variational estimation of the matrix element. ${ }^{10}$ Both results would require considerable effort.

Equation (2.4) does not represent a unique choice for the modified Hamiltonians. In fact, there exist $^{11}$ alternative modified Hamiltonians which possess the required positive definite properties. However, no numerical studies have been made as yet to to test these latter modified Hamiltonians.

Finally, we comment briefly on some computational aspects of the variational calculation. First, the calculation of an auxiliary Lagrange function requires inversion of a matrix in contrast with the diagonalization of the matrix required in the determination of the wave functions. Thus round-off error is less of a problem for the variational calculation than for the direct first-order calculation using more elaborate wave functions. Second, a variational calculation employing a wave function with $N_{\phi}$ parameters and an auxiliary function with $N_{L}$ parameters is more convenient than the one employing a wave function with $N=N_{\phi}+N_{L}$ parameters, since wave functions with fewer parameters $N_{\phi}$ are generally available in the literature. This reduces the problem with $N$ parameters to one with $N_{L}$ parameters. These remarks suggest that the variational procedure may often be more efficient than the use of more elaborate functions, but the question remains open.

\section{ACKNOWLEDGMENTS}

We are indebted to Dr. A. W. Weiss for sending us unpublished material on his ground-state and excited-state wave functions. This research was supported in part by the National Science Foundation under Grant No. PHY 77-10131 and by the Office of Naval Research under Contract No. N00014-76-0317. This work was submitted by one of us (J.M.W.) in partial fulfillment of the requirements for the degree of Doctor of Philosophy at New York University. 
*Present address: Group T-4, Los Alamos Scientific Laboratory, Los Alamos, N.M. 87545.

${ }^{1}$ C. Schwartz, Ann. Phys. (N.Y.) $\underline{6}, 156$ (1959); $\underline{6}, 170$ (1959).

${ }^{2}$ A. Dalgarno and A. L. Stewart, Proc. R. Soc. Lond. A 257, 534 (1960); L. M. Delves, Proc. Phys. Soc. Lond. 92,55 (1967).

${ }^{3}$ E. Gerjuoy, A. R. P. Rau, and L. Spruch, Phys. Rev. A 8, 662 (1973).

${ }^{4}$ E. Gerjuoy, A. R. P. Rau, L. Rosenberg, and L. Spruch, Phys. Rev. A 9, 108 (1974).

${ }^{5}$ R. Shakeshaft, L. Rosenberg, and L. Spruch, Phys. Rev. A 14, 1989 (1976).
${ }^{6}$ J. F. Hart and G. Herzberg, Phys. Rev. 106, 79 (1957). ${ }^{7}$ Y. K. Kim and M. Inokuti, Phys. Rev. 175, 176 (1968).

${ }^{8}$ R. Blau, A. R. P. Rau, and L. Spruch, Phys. Rev. A 8 , 131 (1973); R. Blau, L. Rosenberg, and L. Spruch, Phys. Rev. A 10, 2246 (1974).

${ }^{9}$ R. Blau, Phys. Rev. A 14, 890 (1976).

${ }^{10} \mathrm{E}$. Gerjuoy, J. Math. Phys. 16, 761 (1975); S. T. Epstein, J. Math. Phys. 18, 342 (1977).

${ }^{11} \mathrm{E}$. Gerjuoy, L. Rosenberg, and L. Spruch, J. Math. Phys. 16, 455 (1975).

${ }^{12}$ C. L. Pekeris, Phys. Rev. 112, 1649 (1958); 115, 1216 (1959); B. Schiff, H. Lifson, C. L. Pekeris, and P. Rabinowitz, Phys. Rev. 140, A1104 (1965). 